\title{
Eficiencia y productividad del cultivo de frijol en un sistema de riego por bombeo en Zacatecas, México
}

\author{
José Luis Ríos Flores*, Miriam Torres Moreno**, Marco Antonio Torres Moreno** y Jesús Enrique Cantú Brito****
}

Recepción: 22 de junio de 2016

Aceptación: 30 de noviembre de 2016

*Universidad Autónoma Chapingo-Unidad Regional Universitaria de Zonas Áridas, México.

**Sagarpa-Región Lagunera-Subdelegación de Planeación y Desarrollo Rural Durango, México.

***Innovación Ambiental para la Conservación y Desarrollo Sustentable A. C. Texcoco, México. ****Universidad Autónoma Agraria Antonio Narro, México.

Correos electrónicos: j.rf2005@hotmail.com;

m.torresm2015@gmail.com; atm.marco@gmail.com;

drcantuje@yahoo.com.mx

Se agradecen los comentarios de los árbitros de la revista.

Resumen. La escasez de agua es un factor limitante para la agricultura. Conforme la competencia por este recurso se intensifica, el sector agrícola debe hacer un uso más eficiente. Se estima la productividad del agua en frijol (Phaseolus vulgaris L.) producido en Calera y Villa de Cos, Zacatecas, con base en la metodología establecida por Ríos et al. (2015). La productividad física fue $0.118 \mathrm{~kg} \mathrm{~m}^{-3}$, es decir, $8442 \mathrm{~L} \mathrm{~kg}^{-1}$ de frijol para el Distrito de Desarrollo Rural 189 Zacatecas. El precio del metro cúbico para riego fue $\$ 0.48 \mathrm{~m}^{-3} \mathrm{y}$ utilidad bruta MN\$1.88m-3. Se concluye que la productividad agrícola del agua en frijol fue baja, por lo que la producción de frijol en bombeo es insostenible.

Palabras clave: agua virtual, huella hídrica, Phaseolus vulgaris L.

\begin{abstract}
Efficiency and Productivity of the Dry Bean Cultivation in an Irrigation System by Pumping, in Zacatecas, Mexico

Abstract. Water scarcity is a limiting factor for agriculture. As the competition for this resource intensifies; the agricultural sector must make a more efficient use. This work estimated the production of water in beans (Phaseolus vulgaris L.) produced in Calera and Villa de Cos, Zacatecas based on the methodology established by Ríos et al., (2015). The physical productivity was $0.118 \mathrm{~kg} \mathrm{~m}^{-3}$, namely; 8 , $442 \mathrm{~L} \mathrm{~kg}^{-1}$ of bean for Rural Development District 189 Zacatecas. The price of the cubic meter for irrigation was $\$ 0.48 \mathrm{~m}^{-3}$ and gross profit MN\$1.88 $\mathrm{m}^{-3}$. It is concluded that the agricultural production of water in beans was low, so the production of beans in pumping is unsustainable.
\end{abstract}

Keywords: virtual water, water footprint, Phaseolus vulgaris L.

\section{Introducción}

El crecimiento del sector agrícola en el pasado se basaba principalmente en la expansión de las áreas de cultivo, tanto de riego como de secano. Sin embargo, en la actualidad el potencial para el crecimiento continuo ha disminuido, dado que la frontera agrícola se ha reducido por la urbanización o la escasez del agua (Molden et al., 2001a). En regiones de escasez de agua de igual manera se está incrementando la competencia por este elemento entre los sectores agrícolas, domésticos, industriales, comerciales y ambientales, lo que ejerce un efecto negativo sobre los recursos hídricos en muchas regiones del mundo. Esta escasez afecta sobre todo a la agricultura, considerada el principal usuario porque utiliza 75\% del total de agua disponible, por lo que la estrategia clave se centra en mejorar la productividad del agua (Molden et al., 2001b).

El concepto de la productividad del agua fue establecido por Kijne et al. (2003) como una medida sólida para determinar la capacidad de los sistemas agrícolas de convertir 
el agua en alimento. Sin embargo, la determinación de tal concepto en la práctica se utiliza como una herramienta de diagnóstico para resolver la eficiencia del uso del agua en los sistemas agrícolas y proporciona una visión sólida para decretar las oportunidades de redistribución de agua en las cuencas, donde el objetivo sea el incremento de la productividad agrícola del agua.

Lo anterior permitirá a los tomadores de decisiones hacer juicios acerca de qué alternativas existen para resolver los problemas técnicos en cuanto a la productividad del agua, o acerca de si una región es o no eficiente en la producción agrícola. El análisis de este tipo de indicadores no debe ser interpretado como un elemento aislado: es una herramienta orientada a brindar información básica que, siendo analizada en el contexto regional y junto con otros indicadores de relevancia, puede ser de utilidad para los tomadores de decisiones (UN-Water, 2012). Los otros factores a considerar son climáticos, hidrológicos y geográficos, así como los modelos productivos utilizados en las distintas regiones, la evolución demográfica local y los escenarios futuros (Mekonnen y Hoekstra, 2010) que contribuyan a disminuir de la presión que se ejerce sobre los recursos hídricos (Aldaya et al., 2010; Hoekstra, 2008; Hoekstra y Chapagain, 2007).

En México aproximadamente 14\% del frijol se produce bajo condiciones de riego, en particular en la región centronorte. Durante el ciclo agrícola 2014, el estado de Zacatecas registró un total de 596944 ha de frijol que representa 34\% de la superficie nacional. En el DDR (Distrito de Desarrollo Rural) 189 Zacatecas se ubican los municipios de Calera, Villa de Cos y Zacatecas que contribuyen con $46.5 \%$ de la producción de frijol establecido bajo condiciones de riego en el estado de Zacatecas (SIAP, 2014).

El Dof (Diario Oficial de la Federación) (2015) indica que del volumen total de agua extraído en el municipio de Calera, $79.37 \%$ (99.21 $\mathrm{Mm}^{3}$ año $\left.{ }^{-1}\right)$ se destina a la agricultura, 15.8\% (19.75 $\mathrm{Mm}^{3}$ año $^{-1}$ ) para uso público urbano, $0.03 \%\left(0.04 \mathrm{Mm}^{3}\right.$ año $\left.{ }^{-1}\right)$ para el sector pecuario y $4.8 \%$ (6.00 $\mathrm{Mm}^{3}$ año $\left.^{-1}\right)$ se emplea en usos industriales, lo cual contribuye con $33.62 \%$ del PIB (Producto Interno Bruto) del estado. Los principales cultivos son el de chile, maíz grano, ajo, cebolla, frijol y cultivos forrajeros (SIAP, 2012). Se aúna a lo anterior las escasas precipitaciones combinadas con la baja eficiencia en el uso del agua, así como las pobres prácticas de manejo en las parcelas que han contribuido con el rápido declive de los niveles en los acuíferos provocando la sobreexplotación donde, de acuerdo con algunos estudios, el déficit de ese acuífero fue $87.57 \mathrm{Mm}^{3}$ (DOF, 2015). Vélez-Rodríguez et al. (2015) mencionan que los acuíferos de Calera y Chupaderos son dos de los más sobreexplotados de la zona hidrográfica administrativa Cuencas Centrales del Norte. Está problemática se agudiza dado que la producción agrícola en estas regiones depende de la disponibilidad del agua, por lo que se debe de analizar cuán productivo es el uso del recurso en esa región. El objetivo de este artículo es entonces determinar la productividad del agua de riego en el cultivo de frijol para el Distrito de Desarrollo Rural 189 Zacatecas, en particular en los municipios de Villa de Cos y Calera.

\section{Materiales y Métodos}

\section{1. Localización del área de estudio}

El clima del Distrito de Desarrollo Rural 189 Zacatecas es del tipo seco templado extremoso (BS1 kw (w) (e) gw") (García, 2004), con una oscilación anual de temperaturas medias mensuales entre 7 y $14{ }^{\circ} \mathrm{C}(\mathrm{e})$. El mes más cálido se presenta antes del solsticio de verano y de la temporada lluviosa (g). Hay dos máximos de lluvias separados por dos estaciones secas, una larga en la mitad fría del año y una corta a mitad de la temporada lluviosa (w") (condición canícula), la cual es importante porque ocurre en el periodo de crecimiento de la vegetación (Pineda-Martínez et al., 2007). La precipitación media anual oscila entre 300 a 400 mm y una temperatura media de $12{ }^{\circ} \mathrm{C}$ del mes más frío y $21{ }^{\circ} \mathrm{C}$ del mes cálido (mapa 1).

\section{1. 1. Fuentes de información}

Por medio de los datos base se obtuvieron todas y cada una de las variables: cifras de superficie cosechada, producción física anual, precios por tonelada (dividiendo el valor bruto de la producción entre la producción física anual), reportados por SIAP (2012) para el área de Villa de Cos y Calera, Zacatecas, ciclo agrícola 2012, y los costos por hectárea y número de jornales por hectárea obtenidos del Sistema de elaboración de Costos Agropecuarios en el Módulo Agrícola de FIRA (2012). Las láminas de riego netas empleadas para riego por bombeo fueron de $1.28 \mathrm{~m}$ para el ciclo vegetativo del cultivo calculadas con el programa de D’Riego (2006) versión 1.0 desarrollado por el CENID-RASPA de Inifap (Instituto Nacional de Investigaciones Forestales Agrícolas y Pecuarias).

\section{2. Uso consuntivo del agua subterránea en la pro- ducción de frijol en Zacatecas}

De acuerdo con el SIAP (2012), en el estado de Zacatecas, durante el ciclo agrícola 2012 se cosecharon un total de 1033962.57 ha, de las cuales $47.71 \%$ (493 $308.61 \mathrm{ha}$ ) fue de frijol. Asimismo, a nivel estatal el ingreso generado por la producción agrícola fue de \$12 466186.76 pesos, del cual el 
Cuadro 1. Superficie, producción, valor de la producción, rentabilidad y uso de agua subterránea en el cultivo de frijol (Phaseolus vulgaris L.) en Villa de Cos y Calera en el DDR 189 Zacatecas. Ciclo agrícola 2012.

\begin{tabular}{|c|c|c|c|}
\hline Variable macroeconómica & Villa de Cos & Calera & DDR 189 Zacatecas \\
\hline Superficie cosechada (ha) & 2970.00 & 1345.00 & 11708.40 \\
\hline Producción anual (ton) & 4920.90 & 2690.00 & 20804.70 \\
\hline $\begin{array}{l}\text { Valor Bruto de la Producción } \\
\text { (millones de pesos) }\end{array}$ & $\$ 87.30$ & $\$ 65.90$ & $\$ 431.00$ \\
\hline Rendimiento (ton ha ${ }^{-1}$ ) & 1.657 & 2.000 & 1.777 \\
\hline Precio/ton & $\$ 17733$ & $\$ 24500$ & $\$ 20716$ \\
\hline Ingreso/ha & $\$ 29380$ & $\$ 49000$ & $\$ 36810$ \\
\hline Costo/ha & $\$ 15945$ & $\$ 15945$ & $\$ 15945$ \\
\hline Ganancia/ha & $\$ 13435$ & $\$ 33055$ & $\$ 20865$ \\
\hline Relación beneficio/costo & 1.84 & 3.07 & 2.30 \\
\hline Lámina neta de riego & 1.50 & 1.50 & 1.50 \\
\hline Volumen de agua (millones de $\mathrm{m}^{3}$ ) & 44.55 & 20.18 & 175.63 \\
\hline $\begin{array}{l}\text { Ganancia monetaria total } \\
\text { (millones de pesos) }\end{array}$ & $\$ 39.90$ & $\$ 44.46$ & $\$ 244.29$ \\
\hline $\begin{array}{l}\text { Capital invertido en la producción } \\
\text { (millones de pesos) }\end{array}$ & $\$ 47.36$ & $\$ 21.45$ & $\$ 186.69$ \\
\hline \multicolumn{4}{|c|}{ Comparativo a nivel estatal } \\
\hline & \multicolumn{2}{|c|}{ Superficie } & Bruto de la Producción \\
\hline Todos los cultivos & \multicolumn{2}{|c|}{1033962.70} & $\$ 12466186.76$ \\
\hline Frijol & \multicolumn{2}{|c|}{493308.61} & $\$ 3649968.36$ \\
\hline Porcentaje & \multicolumn{2}{|c|}{$47.71 \%$} & $29.28 \%$ \\
\hline
\end{tabular}

Fuente: elaboración propia con base en cifras del SIAP (2012) y FIRA (2012). frijol aportó 29.27\% (MN\$3 649968.36 pesos) de ese valor bruto de la producción agrícola. Desglosando esas cifras, se encontró que el DDR 189 Zacatecas contribuyó con 11708.4 ha de frijol, de las 493308.61 ha cosechadas en el estado, es decir, contribuyó con $2.37 \%$ de la superficie de frijol cosechada en el estado. Desglosando esas cifras, se observa que en Calera se cosecharon 1345.0 ha, lo que representó $11.48 \%$ de la superficie establecida con frijol en el DDR 189 Zacatecas, mientras en Villa de Cos se cosecharon 2970.0 ha, lo que representó $25.36 \%$ de la superficie cosechada del DDR 189, Zacatecas (cuadro 1).

Asimismo, de acuerdo con el cuadro 1 la producción de frijol en el DDR 189 Zacatecas fue de 20804.7 toneladas de frijol, de las cuales Villa de Cos aportó $23.65 \%$ de esa producción, mientras

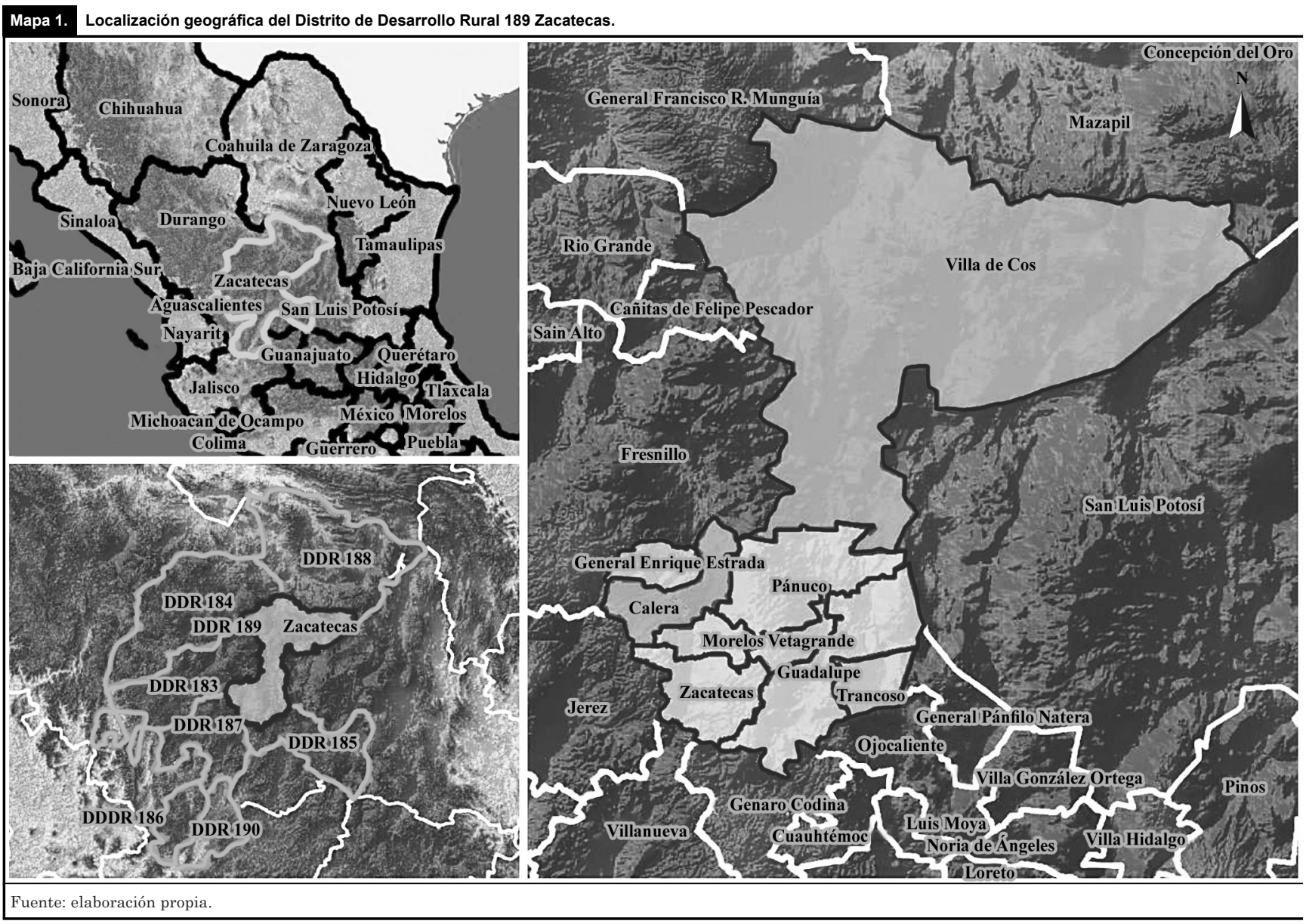


que Calera aportó 2690 toneladas, lo que representó 12.92\% de la producción de ese DDR 189 Zacatecas. Esa producción en el DDR 189 Zacatecas generó un valor de la producción igual a MN\$431 millones de pesos, de los cuales la región de Villa de Cos aportó 20.25\%, mientras que Calera aportó $15.29 \%$.

En este tenor, en el DDR 189 Zacatecas, las 11708.4 ha cosechadas de frijol emplearon una lámina de riego neta de $1.50 \mathrm{~m}$, por lo que a nivel regional el volumen total de agua subterránea empleada para el riego de frijol ascendió a 1755.63 millones de metros cúbicos $\left(\mathrm{Mm}^{3}\right)$, de los cuales el municipio de Villa de Cos empleó $44.50 \mathrm{Mm}^{3}$, lo que representó $25.33 \%$ del total, mientras en Calera se emplearon 20.18 $\mathrm{Mm}^{3}$, lo que representó $11.48 \%$ del total del agua subterránea empleada en el DDR 189 Zacatecas.

\section{3. Indicadores de eficiencia y productividad agrícola del agua}

Se emplearon modelos matemáticos para estimar la productividad del agua de riego en términos físicos $\left(Y_{1}\right.$ y $\left.Y_{2}\right)$, económicos $\left(Y_{3}-Y_{7}, Y_{12}-Y_{13}\right)$ y sociales $\left(Y_{8}-Y_{11}\right)$, diseñados por Ríos et al. (2015).

La variable $Y_{1}$ muestra la relación entre el volumen $(V)$ de agua que representa la demanda hídrica del cultivo en una hectárea $\left(\mathrm{en}^{3}\right)$ y el rendimiento físico $(R F)$ por hectárea (en $\mathrm{kg}$ ); $V$ es el producto de $10000 \mathrm{~m}^{2}$ por la lámina de riego $(L R)(\mathrm{en} \mathrm{m})$ dividida entre el coeficiente de eficiencia de riego $(E C)$ (en porcentaje, en base 1, considerando la relación que existe entre el volumen de agua suministrado y volumen de agua realmente aplicado al cultivo). Se expresa en metros cúbicos de agua empleados en el riego por kilogramo de frijol producido, mientras que su forma inversa daría origen a la variable $Y_{2}$ y se expresaría en kilos por metro cúbico $\left(\mathrm{kg} \mathrm{m}^{-3}\right)$.

$$
\begin{aligned}
& Y_{1}=\frac{V}{R F}=\frac{10000\left(\frac{L R}{E C}\right)}{R F}=10^{4}(L R)(E C)(R F)^{-1} \\
& Y_{2}=\frac{R F}{10000\left(\frac{L R}{E C}\right)}=\left(10^{-4}\right) R F(L R / E C)^{-1}
\end{aligned}
$$

La variable $Y_{3}$ muestra la relación entre la cantidad de agua empleada en el riego $(V)$ y el ingreso o rendimiento mone-

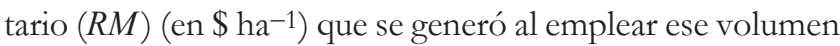
de agua, donde el numerador de esta ecuación es el mismo numerador de $Y_{1}$ y el denominador $(R M)$ es igual al producto de $(R F)$ por el precio $(p)$ (en $\$ \mathrm{~kg}^{-1}$ ). Se expresa en metros cúbicos por peso MN\$ de ingreso bruto producido por el cultivo. Por otro lado, la forma inversa de $Y_{3}$ (la variable $Y_{4}$ ) se interpretará como la cantidad de ingreso monetario generado por el cultivo por cada metro cúbico de agua irrigada en la producción (en $\mathrm{MN} \$ \mathrm{~m}^{-3}$ ).

$$
\begin{aligned}
& Y_{3}=\frac{V}{R M}=\frac{10000\left(\frac{L R}{E C}\right)}{R F(p)}=10^{4}\left(\frac{L R}{E C}\right)\left(R F^{-1}\right)\left(p^{-1}\right) \\
& Y_{4}=\frac{R M}{V}=\frac{R F(p)}{10000\left(\frac{L R}{E C}\right)}=10^{-4} R F(p)\left(\frac{L R}{E C}\right)^{-1}
\end{aligned}
$$

En la variable $Y_{5}$ se representa la relación entre la utilidad monetaria o ganancia $(U)$ y el volumen $(V)$ de agua empleado. El numerador es la utilidad bruta $(U)$ generada por el cultivo, la cual es igual a la diferencia entre el ingreso monetario por hectárea $(R M)$ y el costo por hectárea $(C)$. El denominador de esta ecuación es el mismo numerador que se señala en la ecuación $Y_{1}$. Esta variable es expresada en MN\$ generados de ganancia por metro cúbico de agua irrigada, mientras que su forma inversa $\left(Y_{6}\right)$ expresa la cantidad de metros cúbicos necesarios para producir MN\$1 de utilidad.

$$
\begin{aligned}
& Y_{5}=\frac{U}{V}=\frac{R M-C}{10000 \frac{L R}{E C}}=\frac{R F(p)-C}{10000 \frac{L R}{E C}} \\
& Y_{6}=\frac{10000\left(\frac{L R}{E C}\right)}{U}=10000\left(\frac{L R}{E C}\right)(R F(p)-C)^{-1}
\end{aligned}
$$

La variable $Y_{7}$ es la división de $Y_{6}$ entre el costo que tiene el metro cúbico de agua $\left(\mathrm{C} \mathrm{m}^{-3}\right)$ para el productor. Expresada como un índice positivo mayor, menor o igual a la unidad, es adimensional. Donde mayor a 1 indica que la utilidad generada por $\mathrm{m}^{3}$ es superior a lo que el productor pagó por cada metro cúbico de agua, y menor a 1 señala que la utilidad generada es inferior al costo del agua.

$$
Y_{7}=\frac{Y_{6}}{C m^{-3}}
$$

La variable $Y_{8}$ determinó el punto de equilibrio $(P E)$, igual a la división del costo por hectárea $(C)$ entre el precio por tonelada $(p)$ señalado en ecuaciones 2 y 3 . Lo anterior tiene su sustento en considerar que el $P E$ es el punto donde el ingreso monetario por hectárea $R M=R f(p)$ es igual al costo por hectárea $(C)$, por lo que al igualar $R F(p) \operatorname{con} C$ y despejar $R F$ queda tal cual se señala la $Y_{8}$. Se expresa en toneladas por hectárea.

$$
Y_{8}=P E=\frac{C}{p}
$$




\section{Resultados y discusión}

\section{1. Índices de productividad física y económica}

El cuadro 2 muestra cada uno de los conceptos componentes del costo total por hectárea del cultivo de frijol, tanto en términos absolutos (MN\$) como en porcentajes (costo relativo). Estos últimos señalan la importancia de cada componente. De esa fuente se observa que el concepto del riego (MX\$7 163) representa 44.9\% del costo total de producción por hectárea (MN\$15 945), mientras que el concepto de siembra y fertilización (MX\$3 460) representa $21.7 \%$. El agua empleada para el riego del cultivo es importante en tanto que su aporte limita la producción de alimentos, generación de energía y las actividades en otros sectores económicos (Gorre-Dale, 1992). Sin embargo, el precio que pagan los productores por metro cúbico (MN\$0.48 $\mathrm{m}^{-3}$ ) fue bajo para el riego de este cultivo (cuadro 2). De allí que surja la pregunta de ¿qué pasará en el futuro de mantenerse ese precio en el agua de riego? Indudablemente el bajo costo promueve el uso irracional del recurso. En el norte de México es común que los productores apliquen riegos rodados o de inundación con base en su experiencia aun cuando cuenten con sistemas de riego presurizado dificultando la correcta aplicación de las láminas de riego recomendadas para cada cultivo, por lo que el establecimiento de un precio que valore al recurso agua como recurso finito promoverá un uso racional.

El precio del agua en la agricultura es importante en tanto que este se relaciona con la demanda del recurso, así como con su conservación de acuerdo con Takele y Kallenbach (2001). Por ello, la valoración monetaria de un metro cúbico de agua es necesaria, pues hace posible comparar y así inferir si es caro o barato ese precio por metro cúbico. En nuestro país, la información respecto del costo del metro cúbico de agua usado en la agricultura es limitada, pues no se le ha dado

Cuadro 2.

Costo por hectárea en el cultivo de frijol (Phaseolus vulgaris L.) producido en el DDR 189 Zacatecas. Ciclo agrícola 2012. Cifras en moneda nacional MN\$.

\begin{tabular}{lccc}
\hline \multicolumn{1}{c}{ Concepto } & Costo absoluto $(\$)$ & Jornales por hectárea & Costo relativo (\%) \\
\hline Preparación del suelo & $\$ 2050.00$ & 0.5 & $12.9 \%$ \\
Siembra y fertilización & $\$ 3460.00$ & 0.1 & $21.7 \%$ \\
Labores de cultivo & $\$ 700.00$ & 0.0 & $4.4 \%$ \\
Riego & $\$ 7163.00$ & 0.2 & $44.9 \%$ \\
Fitosanidad & $\$ 996.00$ & 6.0 & $6.2 \%$ \\
Cosecha & $\$ 900.00$ & 0.1 & $5.6 \%$ \\
Diversos & $\$ 253.00$ & 1.3 & $1.6 \%$ \\
Costo financiero & $\$ 423.00$ & 8.2 & $2.7 \%$ \\
Costo total por hectárea & $\$ 15945.00$ & & \\
Precio del ${ }^{3}$ de agua & $\$ 0.48$ & & \\
\hline Fuente: elaboración propia con base en cifras de FIRA (2012). & & \\
\hline
\end{tabular}

la importancia debida por la escasez del recurso hídrico. El cuadro 2 muestra que en el DDR 189 Zacatecas, el m ${ }^{-3}$ de agua irrigada tuvo un precio de MX\$0.48, mientras que ese mismo volumen de agua en la producción de trigo en el Valle de Mexicali y Ensenada, Baja California, de acuerdo con Ríos et al. (2016), tuvo un precio de $\mathrm{MN} \$ 0.19 \mathrm{~m}^{-3}$. Asimismo, Ríos et al. (2015) determinaron que en la Comarca Lagunera el precio del agua para uso agrícola fue de MN\$0.36 $\mathrm{m}^{-3}$ en promedio, oscilando desde MN\$0.42 $\mathrm{m}^{-3}$, en el ryegrass hasta MN\$0.32 $\mathrm{m}^{-3}$ en avena forrajera, por lo que contra esos precios del agua en el mismo norte de México los MX\$0.48 $\mathrm{m}^{-3}$ del frijol de Zacatecas aparecerían como un precio caro. No obstante, esos mismos MX\$0.48 $\mathrm{m}^{-3}$ al contrastarles con el precio del agua en otras regiones agrícolas del mundo.

De acuerdo con Gleick (2004), agricultores de los Estados Unidos de América pagan entre US\$0.01 a US\$0.05 m-3, mientras que la población en general paga US\$0.30 a US\$0.80 $\mathrm{m}^{3}$ por agua tratada de uso doméstico. Pimentel et al. (2004) reportaron para Israel precios de US\$0.57 (igual a MX\$10.7 a paridad cambiaria actual) en el cultivo de tomate y US\$0.13 $\mathrm{m}^{-3}$ (equivalente a MX\$2.42 a paridad cambiaria actual) en maíz grano, lo que indicaría que en el estado de Zacatecas, donde el agua subterránea tiene un costo de solamente MX\$0.48 $\mathrm{m}^{-3}$ (equivalente a US\$0.02) el precio del agua es en extremo barato, y se promueve así la ineficiencia en el uso del agua, dado que si un bien tiene un precio cercano a cero, la teoría económica muestra que ante los ojos del consumidor de ese bien aparecerá como un bien infinitamente abundante; por lo tanto, le restará importancia y promoverá su uso ineficiente e indiscriminado.

En diferentes escalas, que van desde la parcela, región, distrito de riego, cuenca hidrográfica, tanto la eficiencia física como la económica tienen diferentes implicaciones y debe ser representadas en diferentes formas. La eficiencia del riego física a escala de cuenca puede ser representada como la relación entre el consumo de agua de riego y la producción en biomasa que se generó al emplear esa cantidad de agua, mientras que la eficiencia económica del uso del aguade riego se refiere a los beneficios económicos generados al emplear esa cantidad de agua para la producción agrícola (Cai et al., 2003).

El análisis de la eficiencia del agua se observa en el cuadro 3, el cual muestra los indicadores productivos, económicos y sociales en el cultivo de frijol. El uso eficiente del agua es uno de los índices más empleados en una gran 
variedad de cultivos en España (García et al., 2013; Lorite et al., 2012; Romero et al., 2006); sin embargo, en México existe muy poca información para algunos cultivos y para otros no existe ningún antecedente al respecto, lo que dificulta las comparaciones entre cultivos. Por esta razón, en nuestro país se deben de realizar estudios que consideren la productividad agrícola con el fin de planear en el país qué regiones son propicias para la producción de determinados productos en función de maximizar las utilidades, la generación de empleo y la producción, al mismo tiempo que se disminuya la cantidad de agua que se empleó para lograr tales objetivos.

En este artículo el indicador de eficiencia física del cultivo producido en Calera fue de $0.133 \mathrm{~kg} \mathrm{~m}^{-3}\left(Y_{1}\right)$. Se encontró un índice menor frijol producido en Villa de Cos, con 0.110 $\mathrm{kg} \mathrm{m}^{-3}$, lo que muestra una menor eficiencia de este cultivo para convertir el agua en biomasa, ya que empleó un total de $9053 \mathrm{~L} \mathrm{~kg}^{-1}\left(Y_{2}\right)$ en comparación con el cultivo de frijol producido en Calera, que empleó $7500 \mathrm{~L} \mathrm{~kg}^{-1}$, mientras que el DDR 189 Zacatecas requirió un total de $8442 \mathrm{~L} \mathrm{~kg}^{-1}$ (gráfica 1). Estos datos son consistentes con los determinados por Kadyampakeni et al. (2013), quienes determinaron un indicador de $0.15 \mathrm{~kg} \mathrm{~m}^{-3}$ para el cultivo de frijol bajo riego por gravedad en Malawi; sin embargo, son bajos en relación con los determinados por López et al. (2011), quienes precisaron un indicador igual a $0.93 \mathrm{~kg} \mathrm{~m}^{-3}$, mientras que
González et al. (2014) para el frijol producido en condiciones de secano en Cuba encontraron índices que oscilaron entre 0.6-1.91 $\mathrm{kg} \mathrm{m}^{-3}$, lo que indica que la productividad del agua es variable entre cultivos y entre regiones.

Estas diferencias se deben a las diversas condiciones climáticas en que se realiza la producción, a las diferencias en la eficiencia en la aplicación del riego, así como al tipo de riego que se emplea (Aldaya et al., 2010). Estos indicadores muestran la gran cantidad de agua que se requiere para producir un kilogramo de producto $8442 \mathrm{~L} \mathrm{~kg}^{-1}$ en promedio. En general, los cereales y leguminosas tienen indicadores de productividad del agua muy bajos; al respecto Oweis y Hachum (2003) encontraron indicadores para los cultivos de lenteja $0.57 \mathrm{~kg}$ $\mathrm{m}^{-3}$, garbanzo $0.61 \mathrm{~kg} \mathrm{~m}^{-3}$ y haba $0.51 \mathrm{~kg} \mathrm{~m}^{-3}$. La variable $Y_{3}$ indica que en el DDR 189 Zacatecas en promedio se generaron MN\$2.45 de ingreso bruto por metro cúbico de agua empleado en el riego de frijol, y el municipio de Calera 1.33 veces más ingreso, mientras que Villa de Cos fue el menos productivo en el uso del agua al producir $80 \%$ del ingreso bruto que se generó en promedio distrital al emplear la misma cantidad de agua dulce (gráfica 2). En efecto, se determinó que en promedio se requirieron 4081 para producir MN\$1 peso de ingreso, oscilando desde 3061 en Calera hasta 5111 en Villa de Cos $\left(Y_{4}\right)$. A grandes rasgos, el municipio de Villa de Cos resultó poco productivo al emplear 90531 de agua




para producir un kilogramo de frijol y generar sólo \$0.90 de utilidad bruta por metro cúbico invertido en el riego de este cultivo; se empleó 66.9\% más agua que Calera para obtener la misma cantidad de ingreso.

Por otro lado, el municipio de Calera empleó 75001 de agua dulce para producir un kilogramo de frijol y con ello gestar un ingreso de MN\$2.20 por metro cúbico irrigado, lo que indica que fue 2.44 veces más productivo al generar más utilidad por metro cúbico (gráfica 2). Si se toma en cuenta que Villa de Cos empleó un total de $44.55 \mathrm{Mm}^{3}$ para el riego de 2970 ha, entonces se produjo una ganancia de MN\$39.90 millones de pesos, mientras que en Calera se emplearon un total de 20.18 $\mathrm{Mm}^{3}$ para el riego de poco más de 1300 ha, las cuales resultaron en una ganancia de $\$ 44.46$ millones de pesos, es decir, a pesar de tener 2.20 veces más ha y haber empleado 2.20 veces más agua generó únicamente 89.74\% que la ganancia de Calera, Zacatecas. Estos datos nos indican que Villa de Cos fue menos eficiente en la utilización del agua de riego en términos económicos en relación con Calera, Zacatecas.

Este tipo de indicadores cobran importancia cuando analizamos que en condiciones promedio se emplearon 0.015 $\mathrm{Mm}^{3}$ por hectárea de frijol bajo riego por bombeo (175.63 $\mathrm{Mm}^{3}$ / $11708.4 \mathrm{ha}$ ). Con lo anterior se infiere que durante el ciclo agrícola 2012 se emplearon en total para la producción estatal de frijol bajo condiciones de riego un total de 406.71
$\mathrm{Mm}^{3}$; se observó un decremento en los años posteriores con $400 \mathrm{Mm}^{3}$ en 2013 y $353.55 \mathrm{Mm}^{3}$ en 2014. Esta disminución en el empleo del agua de riego se explica en tanto que la superficie de frijol bajo riego ha ido disminuyendo debido a la poca disponibilidad de recurso hídrico y deja $96 \%$ de la producción de frijol en áreas de temporal, donde el aporte hídrico depende en su totalidad de la lluvia, lo que indica que la mayor parte de la producción de frijol en Zacatecas depende de la lluvia que se presente, de allí que los rendimientos observados entre el 2011 y el 2014 fueran de 0.365 a 0.600 kilogramos por hectárea.

En este tenor, se determinó que en promedio se emplearon 7191 de agua dulce para obtener MN\$1 de utilidad oscilando desde 454 l en Calera hasta 11161 en Villa de Cos, y muestra la gran cantidad de agua que se requiere para generar 1 peso de utilidad. La variable $Y_{7}$ arrojo un índice igual a 1.88, lo que sugiere que de cada peso que el productor de frijol pagó por el costo del metro cúbico de agua se obtuvo de retorno ese peso más MN\$0.88 centavos adicionales. En este sentido Al-Karablieh et al. (2012) en un estudio para todo el patrón de cultivos del río Jordán determinaron indicadores para los cultivos de haba US\$0.59, ejote US\$1.58, chícharo US\$0.68 y frijol US\$0.88, en tanto que el costo del agua de riego fue de US\$0.36. Asimismo, para cultivos como el trigo se han determinados índices de utilidad bruta (€0.23 $\left.\mathrm{m}^{-3}\right)$, mientras

Índices de productividad del agua en frijol producido en el DDR 189 Zacatecas.

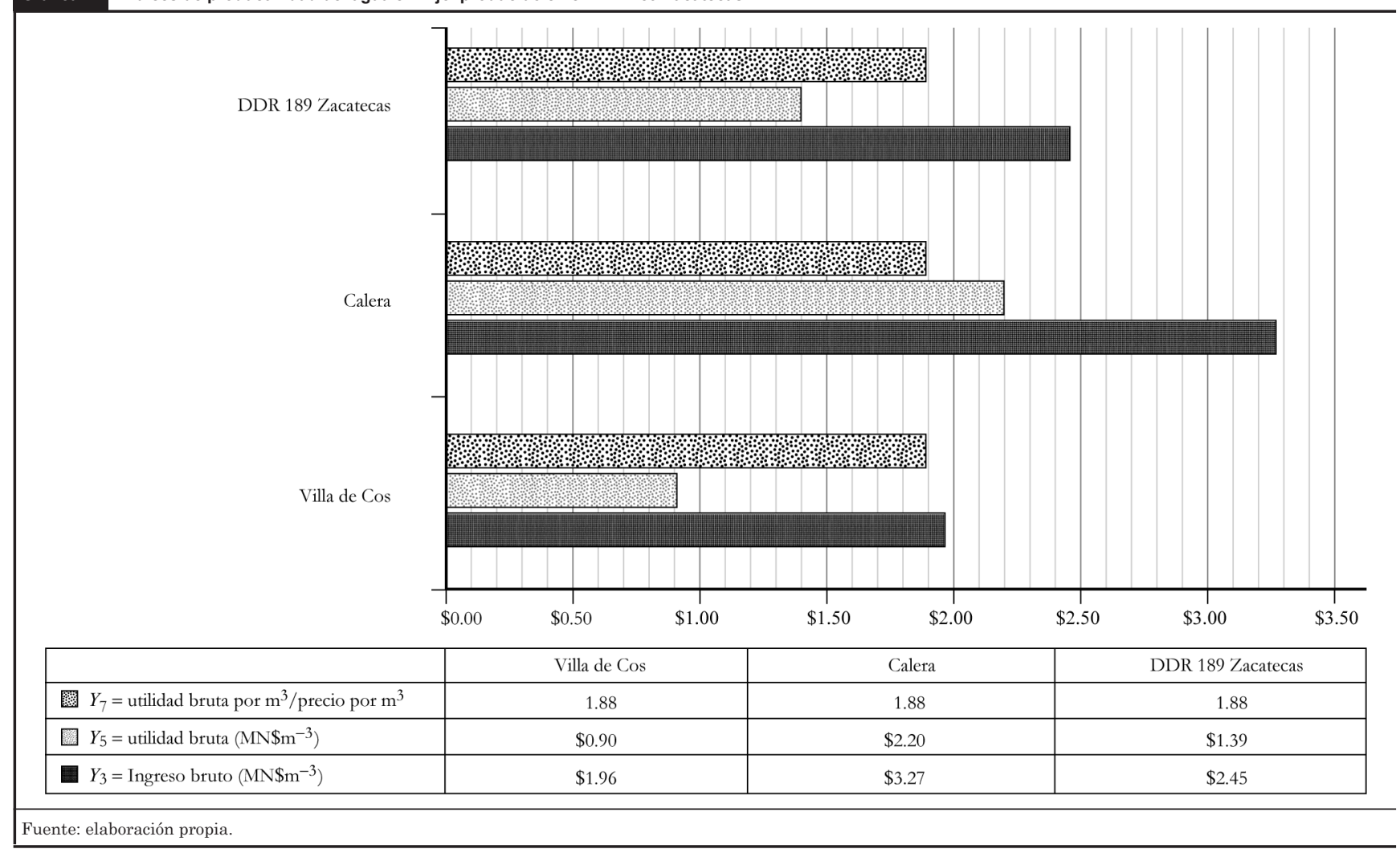


que en girasol y maíz grano fue de $€ 0.53 \mathrm{~m}^{-3}$ (García et al., 2013; Romero et al., 2006), por otro lado Kumar et al. (2011) para la región de Gujarat, India reportan $\mathrm{MN} \$ 2.51 \mathrm{~m}^{-3}$ para el cultivo de frijol.

¿Qué nos indica este valor? más aún ¿para qué nos sirve saber ese dato al compararlo con el determinado en otras regiones? Para lograr entender lo que estos precios implican se presenta la gráfica 3, en ella se indican las tarifas por metro cúbico de agua para uso doméstico para la región de Calera, Zacatecas establecidas por el Sistema de Agua Potable y Alcantarillado en Calera (SAPAC). Esas tarifas se adecúan mensualmente dependiendo de la zonas establecidas por el SAPAC (2015), de esas cifras se puede observar que en promedio para la zona 1 fue de MN\$45.32 y MN\$50.33 para la zona 2 , mientras el precio del metro cúbico para el riego de frijol fue de $\mathrm{MN} \$ 0.48$, aunque el agua de consumo doméstico contempla procesos para hacerla potable la diferencia de precios es dispar de manera notoria, por ello la importancia de determinar la productividad agrícola del agua en los cultivos que conforman el patrón agrícola de determinada región (gráfica 3). Esta información también nos muestra cómo el establecimiento de una tarifa acorde a la realidad de cada región favorecería la eficiencia en la utilización del agua y promovería la generación de más producto por unidad de agua empleada en el riego. Por otra parte, con base en este indicador si se evaluara un grupo de cultivos en esa región podríamos determinar qué cultivos emplean menos cantidad de agua y generan más ingreso, empleos y ganancia en la región, con lo que se podría hacer una planeación en torno a los cultivos que generan más producto en términos físico, económico o social por unidad de agua empleada en el riego.

Sin duda, la producción de alimentos es importante para nuestro consumo, más por tratarse sobre todo de cultivos básicos como el frijol; sin embargo, en las zonas áridas y semiáridas del país, donde el recurso hídrico es escaso, la producción de alimentos se debe planear en función del agua disponible y de un uso racional que garantice la producción de alimentos a través del tiempo y no sólo en la actualidad. En este sentido, la región del altiplano de Zacatecas se encuentra en un punto crítico de fragilidad de sus recursos, ya que 97\% de la producción agrícola depende de la disponibilidad del agua subterránea (Conagua, 2002). Algunos autores mencionan que para mantener la seguridad alimentaria en México se necesitará un incremento de la extracción de agua y de la frontera agrícola (Castro, 2000), lo que pone de manifiesto la vulnerabilidad de la agricultura ante un escenario de crisis del agua.

El caso del mar de Aral es un buen ejemplo del uso irracional del agua dulce para fines agrícolas, que una vez fue

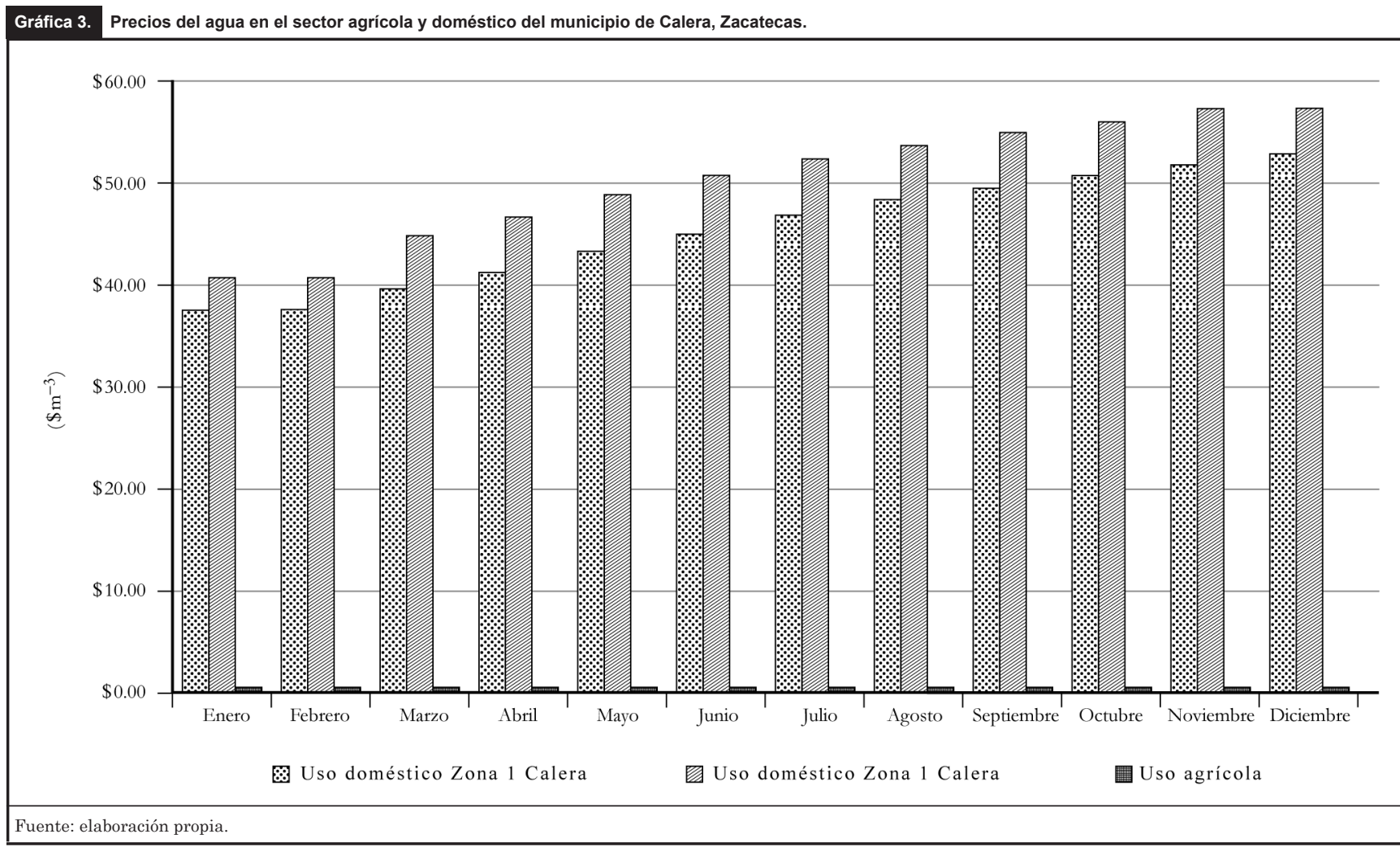


considerado el cuarto lago más grande del mundo. En la actualidad cubre menos de $10 \%$ de lo que fue su superficie hasta los años sesenta del siglo pasado. Esa cuenca soportaba más de 60 millones de personas de seis países diferentes de Asia central: Afganistán, Kazajistán, Kirguistán, Tayikistán, Turkmenistán y Uzbekistán. Las obras de riego empezaron a construirse en 1930, pero tuvieron un repunte importante en el decenio de 1960 cuando los soviéticos decidieron incrementar sustancialmente la producción de algodón y arroz para surtir la creciente demanda interna y exportar el producto. El problema es que el algodón y el arroz emplean gran cantidad de agua para su producción, lo que provocó que con el tiempo el mar redujera su tamaño y volumen contribuyendo al desplome de la industria pesquera y a la afectación del consumo de agua potable. Las zonas secas se convirtieron en cúmulos de polvo y sal y se conformaron desiertos artificiales como el Aral-kum. Estas arenas contaminadas convertidas en tormentas de arena han provocado problemas de salud en la población y a la biodiversidad de los bosques de coníferas que una vez cubrieron superficies mayores a 500000 ha y que en la actualidad se redujeron a 10\% de esa superficie. Los daños fueron incalculables, no sólo en términos ecológicos, sino económicos, sociales, demográficos y políticos.

\section{Análisis prospectivo}

A partir del análisis realizado surgen varias preguntas como ¿qué se puede esperar a futuro con los resultados que se presentan y se discuten? y ¿qué se podría hacer para incrementar la productividad del agua y ser más eficientes en su utilización si se parte de los indicadores determinados? Para responder tales cuestionamientos se evaluaron tres posibles escenarios, que se muestran en el cuadro 3.

Escenario 1. Desaparición del cultivo de frijol bajo condiciones de riego y traslado en su totalidad a condiciones de temporal.

En este escenario se evaluó el efecto que tendría la desaparición del cultivo de frijol bajo riego si se parte de que la disponibilidad del agua para riego en el futuro será menor, aunado a que las áreas de riego se destinarán a cultivos que sean más rentables como las hortalizas, entre las cuales destaca el chile.

En este escenario se consideraron las mismas proporciones observadas en el ciclo agrícola 2012, de tal forma que las 11708 ha de frijol bajo riego se trasladarían a producción de secano o temporal. En términos generales, de realizarse está reconversión el análisis indica que la producción de frijol de las 11708 ha descendería 69\% (el indicador fue
0.31) debido a la caída que tendrían en su rendimiento. No obstante en términos de volumen de agua empleado para la producción de frijol, descendería $74 \%$ (el indicador fue 0.26), al pasar de $175.63 \mathrm{Mm}^{3}$ que están empleados a $44.80 \mathrm{Mm}^{3}$. En este punto es importante mencionar que los $44.80 \mathrm{Mm}^{3}$ de agua que se emplearían por las poco más de 1100 ha provendrían de la lluvia, es decir, no se extraería agua subterránea para la producción, y se utilizaría únicamente el agua de lluvia que de otra forma se perdería por la evaporación, infiltración y percolación. El análisis de este escenario sugiere que la productividad física del frijol se elevaría al disminuir la cantidad de agua empleada por kilogramo producido al pasar de $8442 \mathrm{~L} \mathrm{~kg}^{-1}$ a 6956 $\mathrm{L} \mathrm{kg}^{-1}$; asimismo la productividad del agua en términos de kilogramos de frijol producidos por metro cúbico de agua incrementaría de $0.118 \mathrm{~kg} \mathrm{~m}^{3}$ a $0.144 \mathrm{~kg} \mathrm{~m}^{-3}$.

Por otro lado, en términos económicos el análisis apunta a que este traslado de la producción de frijol de riego a temporal tendría un efecto adverso en la productividad económica del cultivo toda vez que la utilidad bruta generada por metro cúbico decrecería $30.2 \%$ al pasar de $\$ 1.39 \mathrm{~m}^{-3}$ a $-\$ 0.42 \mathrm{~m}^{-3}$, es decir, la producción de frijol en temporal estaría generando una pérdida en este indicador, aun cuando el ingreso bruto obtuvo un indicador positivo igual a $\$ 1.77 \mathrm{~m}^{-3}$, que fue $28 \%$ menor al obtenido en el escenario actual $\$ 2.45 \mathrm{~m}^{-3}$. La caída en la productividad económica del frijol producido bajo este escenario está relacionado de manera directa a la diferencia de precios entre la producción de riego y de temporal $(\$ 20$ 716 ton $^{-1}$ versus $\$ 12278$ ton $^{-1}$ ).

Con esto se puede pronosticar que, en efecto, ante la falta de agua, los agricultores decidirán establecer en las áreas de riego aquellos cultivos que les sean más redituables, aunque está decisión sin duda implica cambios no sólo de los paquetes tecnológicos, también implica un problema para los productores que cuentan con toda la infraestructura para la producción de frijol. Así pues, antes de proponer una reconversión de tal magnitud se deben evaluar otros posibles escenarios.

Escenario 2. Tecnificación del riego en 100\% de la superficie cultivable de frijol

Sin duda una de las estrategias para elevar la productividad del agua es la tecnificación y modernización del riego agrícola. De acuerdo con Ugalde-Acosta et al. (2005), para el cultivo de frijol el sistema que mejor se adapta para aumentar la producción de frijol y elevar la productividad del agua es el riego por goteo con una eficiencia de $97.9 \%$.

En este segundo escenario se asume que ante la creciente necesidad de producir alimentos, una de las opciones a desarrollar en los próximos años será utilizar sistemas de riego que eleven la productividad y eficiencia del agua en los cultivos. 
El análisis indica que de tecnificarse $100 \%$ de la superficie de frijol con sistemas de riego por goteo la producción se elevaría 25\% (de 20804.70 ton a 25904.84 ton), dado que el rendimiento por hectárea pasaría de 1.77 hasta 2.21 ton ha-1 (cuadro 3).

El análisis sugiere que el costo de producción del cultivo se elevaría 3.20 veces al pasar de \$15 945 ton $^{-1}$ a \$50 945 ton $^{-1}$, ya que en ese ciclo se tendría que incluir dentro de los costos de producción el del sistema de riego por goteo que tiene un costo de $35000 \mathrm{ha}^{-1}$ a $40000 \mathrm{ha}^{-1}$. Cabe hacer mención que este sistema de riego tiene una vida útil de entre 15 y 20 años con un adecuado manejo del equipo. Al implementar este sistema de riego el volumen de agua para la producción de frijol descendería $15 \%$ al pasar de $175.63 \mathrm{Mm}^{3}$ a 149.28 $\mathrm{Mm}^{3}$. En otras palabras, para ahorrar un total de $26.35 \mathrm{Mm}^{3}$ se tendrían que invertir un total de \$4097.95 millones de pesos para la tecnificación de las más de 11000 ha de frijol.

La productividad del agua se elevaría 32\% al emplear menos 1 por kilogramo de frijol producido, al pasar de 8442 $\mathrm{L} \mathrm{kg}^{-1}$ a $5763 \mathrm{~L} \mathrm{~kg}^{-1}$. Asimismo, la productividad del agua en términos de kilogramos de frijol producidos por metro cúbico de agua incrementaría de $0.118 \mathrm{~kg} \mathrm{~m}^{-3}$ a $0.174 \mathrm{~kg}$ $\mathrm{m}^{-3}$. El análisis económico indica que si el 100\% de las ha se tecnificaran con riego por goteo la utilidad bruta generada por metro cúbico decrecería $29 \%$ al pasar de $\$ 1.39 \mathrm{~m}^{-3}$ a $\$ 0.40 \mathrm{~m}^{-3}$, mientras que el ingreso bruto sería $47 \%$ superior al pasar de $\$ 2.45 \mathrm{~m}^{-3}$ a $\$ 3.59 \mathrm{~m}^{-3}$ debido al incremento en los rendimientos del cultivo.
Por otro lado, es de considerar que la Sagarpa a través del Programa de Fomento a la Agrícultura en su componente Tecnificación de Riego tiene por objetivo mejorar el uso del agua a nivel parcelario en las Unidades Económicas Rurales Agrícolas, el cual dentro de sus conceptos incluye riego por aspersión (pivote central, avance frontal, Side Roll, aspersión fija, aspersión portátil, cañón y cañón viajero, por microaspersión y goteo).

El riego por goteo cuenta con apoyo de hasta \$17000 por hectárea para productores con más de tres hectáreas, y hasta $\$ 20000$ por hectárea para productores con superficies menores o igual a tres hectáreas. De allí que con base en el mismo escenario 2, con apoyo federal para la tecnificación del 100\% de la superficie de frijol tomando la importancia social y alimentaria que tiene el cultivo, se consideró que en promedio cada productor tendría más de tres hectáreas de frijol por lo que el apoyo federal sería de máximo \$17 000 pesos por hectárea ( $\$ 199.04$ millones de pesos para toda la superficie de frijol en riego), lo que favorecería directamente al productor al disminuir los costos por hectárea y elevar la rentabilidad de 0.91 a 1.35 de considerarse o no el apoyo federal.

De acuerdo con el análisis de ambos escenarios pareciera que la tecnificación de la superficie total de frijol pareciera ser la más viable; sin embargo, la inversión que se requeriría con o sin apoyo federal sería cuantiosa. Otra de las ventajas que se tendrían de tecnificar la superficie es el empleo de fertilizantes líquidos a través de la cintilla, lo que incrementaría

\begin{tabular}{|c|c|c|c|c|c|c|c|}
\hline \multirow[b]{2}{*}{ Variable } & \multirow[b]{2}{*}{$\begin{array}{c}\text { Escenario } 1 \\
\text { Disminuir } \\
100 \% \text { de la } \\
\text { superficie de } \\
\text { frijol en riego } \\
\text { y llevarla a } \\
\text { temporal }\end{array}$} & \multicolumn{2}{|c|}{ Escenario 2} & \multirow[b]{2}{*}{$\begin{array}{c}\text { Situación actual } \\
\text { del DDR } 189 \text { en } \\
\text { promedio }\end{array}$} & \multirow[b]{2}{*}{$\begin{array}{l}\text { Escenario } 1 \text { / } \\
\text { situación actual }\end{array}$} & \multirow[b]{2}{*}{$\begin{array}{c}\text { Escenario } 2 / \\
\text { situación actual* }\end{array}$} & \multirow[b]{2}{*}{$\begin{array}{c}\text { Escenario } 2 / \\
\text { situación actual** }\end{array}$} \\
\hline & & $\begin{array}{c}\text { Mantener la su- } \\
\text { perficie actual y } \\
\text { tecnificar } 100 \% \\
\text { de la superficie } \\
\text { con riego por } \\
\text { goteo* }\end{array}$ & $\begin{array}{c}\text { Mantener la su- } \\
\text { perficie actual y } \\
\text { tecnificar } 100 \% \\
\text { de la superficie } \\
\text { con riego por } \\
\text { goteo** }\end{array}$ & & & & \\
\hline Superficie sembrada (ha) & 11708.40 & 11708.40 & 11708.40 & 11708.40 & 1.00 & 1.00 & 1.00 \\
\hline Producción (ton) & 6439.62 & 25904.84 & 25904.84 & 20804.70 & 0.31 & 1.25 & 1.25 \\
\hline Rendimiento (ton/ha) & 0.55 & 2.2125 & 2.2125 & 1.77 & 0.31 & 1.25 & 1.25 \\
\hline Precio/ton & $\$ 12278.74$ & $\$ 20716.00$ & $\$ 20716.00$ & $\$ 20716.00$ & 0.59 & 1.00 & 1.00 \\
\hline Costo/hectárea & $\$ 8359.00$ & $\$ 50945.00$ & $\$ 33945.00$ & $\$ 15945.00$ & 0.52 & 3.20 & 2.13 \\
\hline Relación beneficio/costo & 0.81 & 0.91 & 1.35 & $\$ 2.30$ & 0.35 & 0.40 & 0.59 \\
\hline Volumen de agua $\left(\mathrm{Mm}^{3}\right)$ & 44.80 & 149.28 & 149.28 & 175.63 & 0.26 & 0.85 & 0.85 \\
\hline$L \mathrm{~kg}^{-1}$ & 6956 & 5763 & 5763 & 8442 & 0.82 & 0.68 & 0.68 \\
\hline $\mathrm{kg} \mathrm{m}^{-3}$ & 0.144 & 0.174 & 0.174 & 0.118 & 1.22 & 1.47 & 1.47 \\
\hline Utilidad bruta $\left(\mathrm{MN} \$ \mathrm{~m}^{-3}\right)$ & $-\$ 0.42$ & $-\$ 0.40$ & $\$ 0.93$ & $\$ 1.39$ & -0.30 & -0.29 & 0.67 \\
\hline Ingreso bruto $\left(\mathrm{MN} \$ \mathrm{~m}^{-3}\right)$ & $\$ 1.77$ & $\$ 3.59$ & $\$ 3.59$ & $\$ 2.45$ & 0.72 & 1.47 & 1.47 \\
\hline
\end{tabular}


los rendimientos. Por otro lado las instituciones y centros dedicados a la investigación tendrán que generar variedades con más tolerancia a la sequía a través del mejoramiento genético, por lo que los esfuerzos deben de centrarse en el desarrollo de la ciencia y la tecnología para lograr obtener mayores rendimientos en los cultivos y al mismo tiempo lograr que la producción sea sustentable.

\section{Conclusiones}

En la actualidad el sector agrícola se enfrenta a retos importantes, entre ellos producir cada vez más alimentos para una población que día a día aumenta su número; sin embargo, esta producción deberá desarrollarse sustentablemente asegurando que las próximas generaciones puedan satisfacer sus necesidades. Con todo, el sector agrícola en estos momentos se orienta a producir más a expensas de deteriorar el medio en el que se desarrolla, por lo que de seguir con la misma visión a largo plazo se podrán observar migraciones masivas de personas que buscan agua potable, así como la compactación de áreas agrícolas que tengan acceso al agua dulce. En Zacatecas se verán cada vez más conflictos entre los agricultores y los usuarios domésticos por el agua para satisfacer las necesidades básicas, como se ha venido observando en el Valle del Yaqui, en Sonora, donde los agricultores demandan que el agua no sea entubada para llevarse a la ciudad, porque ese valle depende de la producción de trigo.
En este sentido, la determinación de índices de productividad del agua en los distintos cultivos de cada región será de utilidad para la toma de decisiones acerca de si una región es propicia para producir determinado producto, más aún si ese cultivo se debe producir bajo condiciones de riego o de temporal, como en el caso del frijol que, como se analizó, empleó grandes cantidades de agua para producir un kilo de producto. Aun cuando se observaron variaciones entre regiones, la cantidad de agua empleada es enorme, por lo que la producción de frijol en Zacatecas debería realizarse sólo en zonas de temporal para aliviar la presión ejercida sobre los recursos hídricos subterráneos de esa región.

En términos económicos también se observó que regionalmente se obtiene más ganancia empleando el agua al uso doméstico que a la producción de frijol bajo riego por bombeo. La situación anterior indica que a futuro podría reconvertirse esas áreas de cultivo con otros más productivos o con variedades de frijol con mayores rendimientos y menores láminas de riego, lo que implica un gran reto para las instituciones dedicadas al mejoramiento genético de cultivos. Asimismo, no tener tarifas tan bajas para uso agrícola en comparación con el uso doméstico favorece el uso irracional del agua, de tal forma que el establecimiento de una tarifa acorde con la disponibilidad mensual o anual de acuerdo con las recargas del acuífero podría promover un uso más eficiente del agua en la agricultura a través del empleo de cultivos o variedades más productivas y rentables.

Referencias

Aldaya, M. M., Martínez-Santos, P. y Llamas, M.

R. (2010). Incorporating the water footprint and virtual water into policy: Reflections from the Mancha Occidental Region, Spain. Water Resources Management, 24(5), 941-958.

Al-Karablieh, E. K., Salman, A. Z., Al-Omari, A. S., Wolff, H. P., Al-Assa'd, T. A., Hunaiti, D. A. y Subah, A. M. (2012). Estimation of the economic value of irrigation water in Jordan. Journal of Agricultural Science and Technology, B2(5B), 487-497.

Cai, X., Rosegrant, M. W. y Ringler, C. (2003). Physical and economic efficiency of water use in the river basin: Implications for efficient water management. Water Resources Research, 39(1), 1-12.
Castro, M. C. (2000). La seguridad alimentaria de México en el año 2030. CIENCIA ergo-sum, 7(1), 49-55.

Conagua (Comisión Nacional del Agua). (2002). Determinación de la disponibilidad del agua en el acuifero chupaderos, estado de Zacatecas. Gerencia de Aguas Subterráneas Subgerencia de Evaluación y Modelación Hidrogeológica. México.

D’Riego (2006). Sistema para la estimación de las demandas de agua y obtención de calendarios de riego para cultivos de los Distritos de Riego del país. Versión 1.0 para Windows. CENID-RASPA, Inifap. ISBN 970-43-0049-2.

DOF (Diario Oficial de la Federación) (2015). Actualización de la disponibilidad media anual de agua en el acuífero Calera (3225), estado de Zacatecas. Publicado el 20 de abril del 2015. Disponible en http://www. conagua.gob.mx/Conagua07/Aguasubterranea/pdf/DR_3225.pdf

FIRA (Fideicomisos Instituidos Relacionados con la Agricultura). (2012). Sistema de elaboración de Costos Agropecuarios. Módulo Agrícola de FIRA. Disponible en www. fira.gob.mx

Gorre-Dale, E. (1992). The Dublin statement on water and sustainable development. Environmental Conservation, 19, 181-181.

García, E. (2004). Modificaciones al sistema de clasificación climática de Köppen (5a ed). México: Instituto de Geografía, unam. 
García, J. G., López, F. C., Usai, D. y Visani, C. (2013). Economic assesment and socioeconomic evaluation of water use efficiency in artichoke cultivation. Open Journal of Accounting, 2(2), 45-52.

Gleick, P. H. (2004). Global freshwater resources: Soft-path solutions for the $21 \mathrm{st}$ century. Science, 302, 1524-1528.

González, R. F., Herrera, P. J., López, S. T. y Cid, L. G. (2014). Productividad del agua en algunos cultivos agrícolas en Cuba. Revista Ciencias Técnicas Agropecuarias, 23(4), 21-27.

Hoekstra, A. Y. (2008). Water neutral: Reducing and offsetting the impacts of water footprints. Value of Water Research Report Series 28. Unesco-IHE.

Hoekstra, A. Y. y Chapagain, A. K. (2007). Water footprints of nations: Water use by people as a function of their consumption pattern. Water resources management, 21(1), 35-48.

Kadyampakeni, D. M., Mloza-Banda, H. R., Singa, D. D., Mangisoni, J. H., Ferguson, A., y Snapp, S. (2013). Agronomic and socioeconomic analysis of water management techniques for dry season cultivation of common bean in Malawi. Irrigation Science, 31, 537-544.

Kijne, J. W., Barker, R. y Molden, D. (eds.) (2003). Water productivity in agriculture: Limits and opportunities for improvement. Colombo: International Water Management Institute.

Kumar, M. D., Scott, C. A. y Singh, O. P. (2011). Inducing the shift from flat-rate or free agricultural power to metered supply: Implications for groundwater depletion and power sector viability in India. Journal of Hydrology, 409(1), 382-394.

López, T., Herrera, J., González, F. y Cid, G. (2011). Modelación de la eficiencia del uso del agua en maíz y frijol en diferentes condiciones de suelos y disponibilidad hídrica. Ingeniería Agrícola, 1(1), 24-29.

Lorite, I. J., García-Vila, M., Carmona, M. A., Santos, C. y Soriano, M. A. (2012). Assessment of the irrigation advisory services' recommendations and farmers' irrigation management: a case study in southern Spain. Water resources management, 26(8), 2397-2419.

Mekonnen, M. M. y Hoekstra, A. Y. (2010). A global and high-resolution assessment of the green, blue and grey water footprint of wheat. Hydrology and Earth System Sciences, 14, 1259-1276.

Molden, D., Sakthivadivel, R. y Zaigham, H. (2001a). Basin-level use and productivity of water: Examples from South Asia. Research Report 49. Colombo: International Water Management Institute.

Molden, D., Upali, A., Intizar, H. (2001b). Water for rural development: Backeground paper on water for rural development prepared for the World Bank. Working paper 32. Colombo: International Water Management Institute. Oweis, T. Y. y Hachum, A. Y. (2003). Improving water productivity in the dry areas of west Asia and North Africa, en J. W. Kijne R. Barker y D. Molden (eds.), Waterproductivity in agriculture: Limits and opportunities for improvement (pp. 179-198). Colombo: International Water Management Institute.

Pimentel, D., Berger, B., Filiberto, D., Newton, M., Wolfe, B., Karabinakis, E. y Nandagopal, S. (2004). Water resources: agricultural and environmental issues. BioScience, 54(10), 909-918.

Pineda-Martínez, L. F., Carbajal, N. y MedinaRoldán, E. (2007). Regionalization and classification of bioclimatic zones applying principal components analysis (PCA) in the central-northeastern region of México. Atmosfera, 20(2), 133-146.

Ríos, F. J. L., Torres, M. M., Castro, F. R., Torres, M. M. A. y Ruiz, T. J. (2015). Determinación de la huella hídrica azul en los cultivos forrajeros del DR-017, Comarca Lagunera, México. Revista Facultad de Ciencias Agronómicas, 47(1), 93-107.

Ríos, F. J. L., Torres, M. M., Ruiz, T. J. y Torres, M. M. A. (2016). Eficiencia y productividad del agua de riego en trigo (Triticum vulgare) de Ensenada y Valle de Mexicali, Baja California, México. Acta Universitaria, 26(1), 20-29.
Romero, P., García, J. y Botía, P. (2006). Costbenefit analysis of a regulated deficitirrigated almond orchard under subsurface drip irrigation conditions in Southeastern Spain. Irrigation Science, 24(3), 175-184.

Sagarpa (Secretaría de Agricultura, Ganadería, Desarrollo Rural, Pesca y Alimentación) (2011). Reporte informativo sobre los efectos de la sequía en México. Subsecretaría de Desarrollo Rural de la Secretaria de Agricultura. Disponible en http://www. gob.mx/siap/

SAPAC (Sistema de Agua Potable y Alcantarillado en Calera, Zacatecas). (2015). Ajuste a las tarifas del agua potable. Disponible en http://www.sapac-calera.gob.mx/portal/

SIAP (Servicio de Información Agroalimentaria y Pesquera) (2012). Anuarios estadísticos de la producción agropecuaria. Sagarpa-SIAP. Disponible en http://www.siap.gob.mx/

SIAP (Servicio de Información Agroalimentaria y Pesquera) (2014). Anuarios estadísticos de la producción agropecuaria. Sagarpa-SIAP. Disponible en http://www.siap.gob.mx/

Takele, E. y Kallenbach, R. (2001). Analysis of the impact of alfalfa forage production under summer water-limiting circumstances on productivity, agricultural and growers returns and plant stand. Journal of Agronomy and Crop Science, 187(1), 41-46.

Ugalde-Acosta, F. J., Villar-Sánchez, B., LópezSalinas, E. y Tosquy-Valle, O. H. (2005). Verificación de tecnología para frijol de riego en la región centro del estado de Veracruz, México. Terra Latinoamericana, 23(1), 599-604.

un-Water (2012). The United Nations World Water Development Report: Managing water under uncertainty and risk. World Water Assessment Programmed (WWAP). Paris: Unesco.

Vélez-Rodríguez, A., Padilla-Bernal, L. E. y Mojarro Dávila, F. (2015). Disponibilidad para ahorrar agua de uso agrícola en México: caso de los acuíferos de Calera y Chupaderos. Revista Mexicana de Ciencias Agrícolas, 6(2), 277-290. 